\title{
An Optimal Service Curve Allocation Scheme Based on RSVP Signaling Protocol
}

\author{
Kihyun Pyun and Dong-Ho Cho
}

\begin{abstract}
An optimal service allocation scheme that can be used in RSVP signaling protocol is proposed to admit real-time sessions with deterministic delay bounds as many as possible. The big merit of our scheme is that it operates in the framework of RSVP, which is recommended by the IETF.
\end{abstract}

Index Terms-Deterministic guaranteed service, RSVP, service curve allocation, resource allocation, packet scheduling.

\section{INTRODUCTION}

$\mathbf{I}$ $\mathrm{N}$ the next generation Internet, deterministic guaranteed service that bounds strict end-to-end packet delays without packet loss becomes important to provide high quality for real-time applications [1]. In particular, the IETF proposes to combine guaranteed service with RSVP signaling protocol [2]. Since deterministic service reserves resource in advance and requires expensive implementation, it is important to allocate resource efficiently to admit real-time sessions as many as possible under RSVP [3].

Unfortunately, to our knowledge, there is no study on how to achieve efficient allocation of resource in each router, given a specific signaling protocol such as RSVP. Note that since RSVP operates over heterogeneous routers that adopt different schedulers, it collects and gives only limited information required for allocating resource in each router. Thus, although resource allocation schemes that do not consider a specific signaling protocol, e.g., the scheme proposed in [4], can achieve a very high number of real-time sessions, they cannot be applicable to the network with heterogeneous routers in which only limited information is given by RSVP. The work proposed in [5] touches on RSVP environment. However, it does not focus on how to allocate resource in each intermediate router with only limited information available while satisfying the end-to-end delay requirement.

In this letter, we propose an optimal resource allocation scheme based on RSVP to admit a high number of realtime sessions in heterogeneous routers. Our scheme saves resource on short-time scales from 'low bandwidth, short delay' sessions to utilize especially for 'high bandwidth, long delay' sessions if the scheduler in the router allows decoupled allocation of delay and bandwidth.

The authors are with the Department of Electrical Engineering and Computer Science, Korea Advanced Institute of Science and Technology (KAIST), Republic of Korea (e-mail: \{khpyun,dhcho\}@ comis.kaist.ac.kr).

This work was supported by the Brain Korea 21 Project in 2003.

\section{Guaranteed SERVice AND RSVP}

\section{A. Network Calculus for Guaranteed Service}

Network calculus for the guaranteed service is based on traffic envelope curve and service curve. We say that session $i$ has a traffic envelope function $E(\cdot)$ if the incoming traffic amount from session $i$ to the network during the interval $(s, t]$ is not greater than $E(t-s)$. Let $S(\cdot)$ be a non-decreasing function with $S(u)=0$ for all $u \leq 0$. We say that a service curve $S(\cdot)$ is guaranteed for session $i$ by a router if, for a packet departure time $t$ of session $i$, there exists a time $s$, $s<t$, such that $s$ is the beginning of one of the session's backlogged periods and

$$
r_{\text {out }}(t) \geq r_{\text {in }}(s)+S(t-s)
$$

where $r_{\text {in }}(t)$ and $r_{\text {out }}(s)$ denote the input amount from session $i$ until the time $t$ and the output amount until the time $s$, respectively. Suppose that a session passes through $M$ routers in tandem and the $m$-th router, $1 \leq m \leq M$, guarantees $S_{m}(\cdot)$ to the session. Then, the $M$ routers guarantee $S_{\text {net }}(\cdot)$ to the session where [6]

$$
S_{n e t}(t)=\min \left\{\sum_{m=1}^{M} S_{m}\left(\Delta_{m}\right): \Delta_{m}>0 \text { and } \sum_{m=1}^{M} \Delta_{m}=t\right\} .
$$

Eq. (2) tells that the network service curve is intuitively constructed by concatenating, in an increasing order of slopes, the line segments of the guaranteed service curves. When the routers guarantee $S(\cdot)$ to the session, the end-to-end delay is not greater than the maximum horizontal distance from the traffic envelope curve $E(\cdot)$ to $S(\cdot)$, denoted by $D(E \| S)$ [6]. Specifically,

$$
D(E \| S)=\max _{k: k>0} \min \{\Delta: \Delta>0 \text { and } E(k) \leq S(k+\Delta)\} .
$$

\section{B. RSVP Combined with Guaranteed Service}

RSVP in conjunction with guaranteed service sets up the connection of a session by two phases, advertisement phase and reservation phase [2].

In the advertisement phase, the source of a session sends a PATH message to the destination. The PATH message includes both the traffic specification called TSpec and the advertisement specification called ADSpec. TSpec represents a special traffic envelope function $E(t)=\min (M+p t, b+r t)$, where $b$ is for the burstiness, $r$ for the average traffic rate, $p$ for the peak rate, and $M$ for the maximum packet size allowed from the session. ADSpec carries information generated or modified at each intermediate router, which is used to derive the service 
curve guaranteed by those routers on the connection path. ADSpec has two important fields $C_{t o t}$ and $D_{t o t}$ that are updated at each intermediate router by adding $C$ and $D$ terms exported by the router to the previous value of $C_{t o t}$ and $D_{t o t}$, respectively, where $C$ and $D$ terms represent the ratedependent and the rate-independent deviations from the ideal fluid model in the router and are determined by an adopted scheduler. See [1] [3] for a detailed explanation. We use the notation $C_{t o t}$ and $D_{t o t}$ to indicate the final value received at the destination.

When the PATH message arrives at the destination, the reservation phase is initiated by sending a RESV message from the destination to the source. The RESV message includes $R$ and $S$ fields. The $R$ field represents the reservation rate. If all the routers in the path reserves at least the $R$ rate for the session, the routers guarantee the following linear service curve $S_{n e t}(\cdot)[1]$ :

$$
S_{\text {net }}(t)=R\left(t-V_{\text {tot }}\right)^{+}=\max \left(0, R\left(t-V_{\text {tot }}\right)\right)
$$

where $V_{t o t}=C_{t o t} / R+D_{t o t}$. We also denote $(C / R+D)$ by $V$ for convenient notation. Since the guaranteed end-to-end delay bound $d_{\max }$ is equal to the horizontal distance $D\left(E \| S_{\text {net }}\right)$,

$$
d_{\text {max }}= \begin{cases}\frac{T(p-R)+M}{R}+V_{\text {tot }}, & p \geq R \geq r \\ \frac{M}{R}+V_{\text {tot }}, & R>p \geq r\end{cases}
$$

where $T$ is the inflexion point of $E(\cdot)$, i.e., $(b-M) /(p-r)$. The destination determines $R$ value such that $d_{\max }$ becomes its desired end-to-end packet delay bound. However, since $R$ cannot be less than the average traffic rate $r$, sometimes we have a time margin, which is stored in the $S$ field. If there is no time margin, the $S$ field has zero value. The $S$ field is updated at each router by the previous value in $S$ field minus the used slack amount in the router. We use the notation $s_{t o t}$ to indicate the initial value at the destination. The desired endto-end delay bound becomes $\left(d_{\max }+s_{t o t}\right)$. If there exists a router that cannot reserve $R$ rate for the session, the session is rejected. Otherwise, the session is accepted.

\section{Service Curve Allocation Problem}

Given the TSpec and RSpec, each router reserves its resource to guarantee a service curve. Deadline-based schedulers such as the service curve scheduler [7] [6] can allocate and guarantee non-linear service curves that allow decoupled allocation of bandwidth and delay requirement in an integrated fashion. However, since only concave piecewise linear service curves result in a constant time for computing deadlines [6] [7], we consider the following concave two piecewise linear curve:

$$
S(t)= \begin{cases}R(t-V-s)^{+}, & t<I \\ r t+f, & t \geq I\end{cases}
$$

where $s$ is the amount of the used slack time and $f$ is the parameter we set. Note that if $f$ value is given, $I$ value is determined since $R \geq r$, or vice versa. The inflexion point $I$ separates the service curve in two phases. In the first phase, the service rate $R$ is assigned to achieve a session's delay goal. The service rate $R$ is identical for both PGPS and deadlinebased schedulers to achieve the same delay guarantee. In the

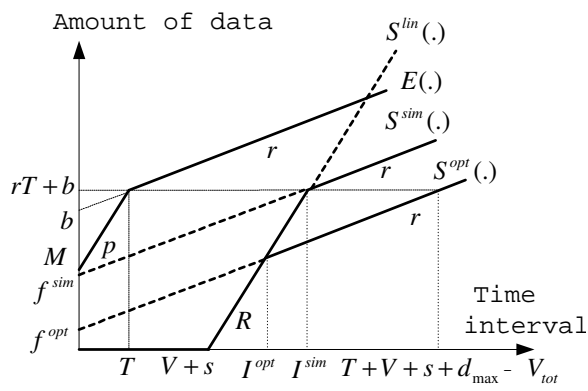

Fig. 1. Different service curves to be guaranteed at the router

second phase, the rate $r$ is assigned to ensure its bandwidth requirement. The sum of the $s$ values in all the routers on the connection path is equal to $s_{t o t}$. Note that routers employing even simple PGPS that usually allocate a linear service curve may also contribute to the improvement of resource efficiency when the proposed scheme is used.

The key problem here is how to choose an optimal parameter $I$ for each session to reserve resource when the RESV message arrives at the respective router. Obviously, the smaller $I$ or $f$ we can choose in (6) the more rate resources we can save for other sessions. Note that, however, the chosen parameter I's at the respective routers must be big enough to satisfy the end-to-end delay requirement. In solving this problem, we have to keep the key constraint due to RSVP; we have to rely on the information that is available in the router. Only eight parameter values are available: four parameters $b, p, r, M$ from TSpec, two parameters $R$ and $s$ from RSpec, and two parameters $C$ and $D$. Note that some important parameter values such as $d_{\max }, s_{t o t}, C_{t o t}$ and $D_{t o t}$ are not known to the router unless the router is the destination.

\section{Proposed Scheme}

A simple solution for satisfying the end-to-end delay requirement is to choose the inflexion point $I^{s i m}=V+s+$ $(r T+b) / R$ as shown in Fig. 1, which results in the following

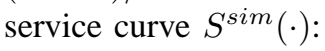

$$
S^{s i m}(t)= \begin{cases}R(t-V-s)^{+}, & t<I^{\text {sim }} \\ r t+f^{s i m}, & t \geq I^{\text {sim }}\end{cases}
$$

where $f^{s i m}=b+r(T-V-s-(r T+b) / R)$. In the figure, $S^{s i m}(\cdot)$ is highly efficient than the linear curve $S^{l i n}(t)=$ $R(t-V-s)^{+}$.

In the case that $R>p$, we can further shift the inflexion point left than $S^{\operatorname{sim}}(\cdot)$. As illustrated in Fig. 1, our idea is to choose the parameter $f$ such that $r(T+V+s+$ $\left.d_{\max }-V_{\text {tot }}\right)+f=E(T)=b+r T$. Note that although we do not know individual values of $d_{\max }$ and $V_{\text {tot }}$, the difference $\left(d_{\text {max }}-V_{\text {tot }}\right)$ becomes $(T(p-R)+M) / R$ if $R \leq p$ from (5). Otherwise, $\left(d_{\max }-V_{t o t}\right)$ is equal to $M / R$. In both cases, $\left(d_{\max }-V_{t o t}\right)$ can be represented by known parameters. Thus, if we denote the chosen $f$ value by $f^{o p t}$,

$$
f^{o p t}= \begin{cases}b-r(V+s+(T(p-R)+M) / R), & R \leq p \\ b-r(V+s+M / R), & R>p .\end{cases}
$$




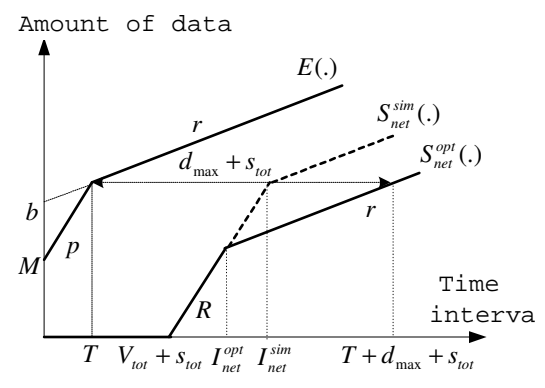

Fig. 2. The guaranteed network service curve in our scheme

Let us denote the specific $I$ value given $f^{o p t}$ by $I^{o p t}$. From (6), $R\left(I^{o p t}-V-s\right)=r I^{o p t}+f^{o p t}$. Thus,

$$
I^{o p t}=V+s+\Delta
$$

where

$$
\Delta= \begin{cases}\frac{b-r(T(p-R)+M) / R}{R-r}, & R \leq p \\ \frac{b-r M / R}{R-r}, & R>p\end{cases}
$$

In summary, the following service curve $S^{o p t}(\cdot)$ is proposed to be guaranteed in the router:

$$
S^{o p t}(t)= \begin{cases}R(t-V-s)^{+}, & t<I^{o p t} \\ r t+f^{o p t}, & t \geq I^{\text {opt }} .\end{cases}
$$

If $R \leq p, I^{o p t}=I^{s i m}$. Otherwise, however, $I^{o p t}-I^{s i m} \leq 0$. Thus, $S^{\text {opt }}(\cdot)$ always reserves smaller or at least the same resources during time intervals than $S^{\operatorname{sim}}(\cdot)$.

Now we show that the proposed scheme satisfies the endto-end delay requirement. In our scheme, each intermediate router guarantees either $S^{o p t}(\cdot)$ if the router allows decoupled allocation of delay and bandwidth or $S^{l i n}(\cdot)$ otherwise. Let us denote the guaranteed network service curve in our scheme by $S_{n e t}^{o p t}(\cdot)$. All the $S^{o p t}(\cdot)$ 's always have the line segment with the slope $R$ during the time interval $\left[V+s, I^{o p t}\right]$ from (11), where $V$ and $s$ usually have different values in different routers. Note that, however, the differences of $\left(I^{o p t}-V-s\right)$ in different routers are all the same $\Delta$ from (9). Thus, from (2), $S_{\text {net }}^{\text {opt }}(\cdot)$ has zero slope until $V_{t o t}+s_{t o t}, R$ slope during the interval $\left[V_{t o t}+s_{t o t}, I_{n e t}^{o p t}\right]$ where

$$
I_{n e t}^{o p t}=V_{t o t}+s_{t o t}+\Delta
$$

and $r$ slope after the time point $I_{n e t}^{o p t}$, as shown in Fig. 2. Specifically,

$$
S_{\text {net }}^{\text {opt }}(t)= \begin{cases}R\left(t-V_{\text {tot }}-s_{\text {tot }}\right)^{+}, & t<I_{\text {net }}^{\text {opt }} \\ r t+f_{\text {net }}^{\text {opt }}, & t \geq I_{\text {net }}^{\text {opt }}\end{cases}
$$

where

$$
f_{\text {net }}^{o p t}= \begin{cases}b-r\left(V_{\text {tot }}+s_{\text {tot }}+(T(p-R)+M) / R\right), & R \leq p \\ b-r\left(V_{\text {tot }}+s_{\text {tot }}+M / R\right), & R>p .\end{cases}
$$

In the figure, $S_{\text {net }}^{\text {sim }}(\cdot)$ and $I_{\text {net }}^{\text {sim }}$ indicate the network service curve and its inflexion point, respectively, if the simple service curve $S^{\operatorname{sim}}(\cdot)$ is guaranteed in each router. We can easily verify that $D\left(E \| S_{n e t}^{o p t}\right)$ becomes exactly $\left(d_{\max }+s_{t o t}\right)$. If we shift more left than $I^{o p t}$, the delay bound requirement is violated. Thus, $S^{\text {opt }}(\cdot)$ is optimal.

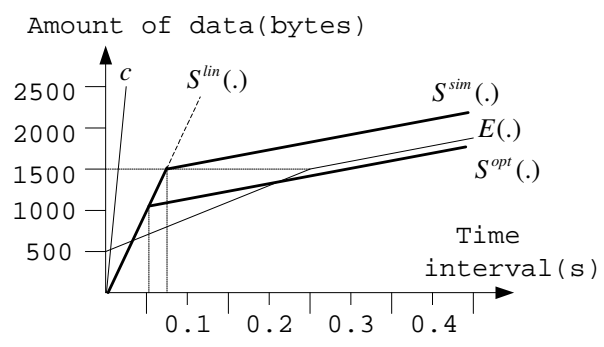

Fig. 3. Different service curves to be guaranteed in the example

\section{NuMERICAL EXAMPLE AND CONCLUSIONS}

Consider a session with $E(t)=\min (500+4000 t, 1000+$ $2000 t$ ) in unit of byte. Let us assume five hops with MTU = 9188 bytes, and link speed $c=155$ Mbps. Suppose that three routers have PGPS schedulers and two routers have service curve schedulers. Each PGPS scheduler exports $C=M$ and $D=M T U / c$. The service curve scheduler exports $C=0$ and $D=M T U / c$. Consequently, we have $C_{t o t}=3 M=$ 1500 bytes and $D_{t o t}=5 \mathrm{MTU} / c=2.371 \mathrm{~ms}$. Let us further assume the destination desires $100 \mathrm{~ms}$ end-to-end delay bound. We then obtain from (5) that $S=0$ and $R=20485$ bytes/s $\approx$ $10 r$. Fig. 3 illustrates the service curve to be guaranteed in our scheme. In the case of the PGPS scheduler, an extremely overprovisioned service curve $S^{l i n}(\cdot)$ is guaranteed inevitably. However, in the case of the service curve scheduler, the optimal service curve $S^{\text {opt }}(\cdot)$ is guaranteed. We have also plotted $S^{\operatorname{sim}}(\cdot)$ for comparison. Note that $I^{o p t}$ and $I^{\text {sim }}$ in this example are located at $52.25 \mathrm{~ms}$ and $74.01 \mathrm{~ms}$, respectively. Thus, $I^{\text {opt }}$ is located more left than $I^{\text {sim }}$ by $21.77 \mathrm{~ms}$.

Note that the saved resource in $S^{o p t}(\cdot)$ can be used especially for 'high bandwidth, long delay' sessions. Thus, we conclude that our scheme can admit a high number of realtime sessions especially when sessions with different delay requirements are mixed.

\section{REFERENCES}

[1] S. Shenker, C. Partridge, and R. Guérin, "Specification of Guaranteed Quality of Service," September 1997, RFC 2212.

[2] J. Wroclawski, "The Use of RSVP with IETF Integrated Services," September 1997, RFC 2210.

[3] J.-Y. L. Boudec and P. Thiran, Network Calculus. A Theory of Deterministic Queueing systems for the Internet. Springer-Verlag, vol. LNCS 2050, ch. 2.

[4] K. Pyun, "Delay-Optimal Service Curve Allocation Scheme," IEEE Communications Letters, vol. 10, no. 7, pp. 505-507, October 2003.

[5] J. Schmitt, "Optimal Network Service Curves Under Bandwidth-Delay Decoupling," Electronics Letters, vol. 38, no. 6, pp. 297-298, March 2002.

[6] K. Pyun, J. Song, and H.-K. Lee, "The Service Curve Service Discipline with the Service Curve Discipline for the Rate-Controlled EDF service discipline in Variable-Sized Packet Networks," in IEEE International Conference on Communications, 2002, pp. 1135-1141.

[7] H. Sariowan, R. L. Cruz, and G. C. Polyzos, "SCED: A Generalized Scheduling Policy for Guaranteeing Quality-of-Service," IEEE/ACM Tran. Networking, vol. 7, no. 5, pp. 669-684, October 1999. 